\title{
Statistics of rigid fibers in strongly sheared turbulence
}

\author{
Dennis Bakhuis, ${ }^{1}$ Varghese Mathai, ${ }^{1,2}$ Ruben A. Verschoof, ${ }^{1}$ Rodrigo Ezeta, ${ }^{1}$ Detlef Lohse, ${ }^{1,3}$ \\ Sander G. Huisman, ${ }^{1}$ and Chao Sun $^{4,1, *}$ \\ ${ }^{1}$ Physics of Fluids Group and Max Planck Center Twente for Complex Fluid Dynamics, MESA+ Institute, \\ University of Twente, Enschede, The Netherlands \\ ${ }^{2}$ School of Engineering, Brown University, Providence, Rhode Island 02912, USA \\ ${ }^{3}$ Max Planck Institute for Dynamics and Self-Organization, Am Faßberg 17, Göttingen, Germany \\ ${ }^{4}$ Center for Combustion Energy, Key Laboratory for Thermal Science and Power Engineering of Ministry \\ of Education, Department of Energy and Power Engineering, Tsinghua University, Beijing, China
}

(Received 24 October 2018; published 15 July 2019)

\begin{abstract}
Practically all flows are turbulent in nature and contain some kind of irregularly shaped particles, e.g., dirt, pollen, or life forms such as bacteria or insects. The effects of the particles on such flows and vice versa are highly nontrivial and are not completely understood, particularly when the particles are finite sized. Here, we report an experimental study of millimetric fibers in a strongly sheared turbulent flow. We find that the fibers show a preferred orientation of $-0.38 \pi \pm 0.05 \pi\left(-68 \pm 9^{\circ}\right)$ with respect to the mean flow direction in high-Reynolds-number Taylor-Couette turbulence, for all studied Reynolds numbers, fiber concentrations, and locations. Despite the finite size of the anisotropic particles, we can explain the preferential alignment by using Jefferey's equation, which provides evidence of the benefit of a simplified point-particle approach. Furthermore, the fiber angular velocity is strongly intermittent, again indicative of point-particle-like behavior in turbulence. Thus large anisotropic particles still can retain signatures of the local flow despite classical spatial and temporal filtering effects.
\end{abstract}

DOI: 10.1103/PhysRevFluids.4.072301

The control and prediction of flows containing anisotropic particles are important for many industrial settings. For example, in the paper production process, the alignment of the fibers of the pulp determines the mechanical strength of the paper [1]. In nature, one objective is on flow prediction, e.g., the dispersion of pollen and seeds [2] or sediment transport in rivers [3,4]. The addition of fibers to the flow can have significant consequences on the rheology of the suspensions $[5,6]$. In homogeneous and isotropic turbulence, rodlike fibers can become preferentially aligned with the vorticity vector [7-11]. Both the fibers and the vorticity are aligned with the largest Lagrangian stretching vector [12]. When the fibers behave as tracers, their orientations become correlated with the local velocity gradients in the flow, and this alignment strongly depends on the fiber shape [10]. In the case of prolate spheroids, the orientation vector is likely to align with the axis of symmetry of the flow [13]. In comparison, the behavior of fibers in viscous shear flows can be noticeably different. Here, the fiber orientation is a result of the competition between alignment by mean velocity gradients and randomization by fluctuating velocity gradients [11]. This can lead to either an alignment parallel to the flow direction [14,15] or at an angle with the wall [16-22]. However, most of the studies in shear flows have been done by numerical simulations, addressing the simplified case of inertial pointlike fibers without gravity. Often a point-particle approach is

*chaosun@tsinghua.edu.cn 
(a)

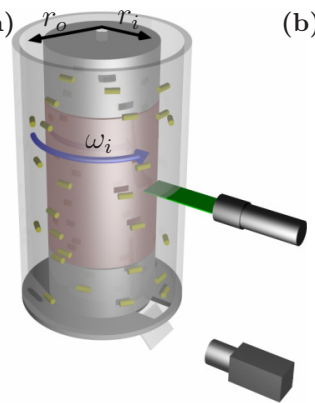

(b)

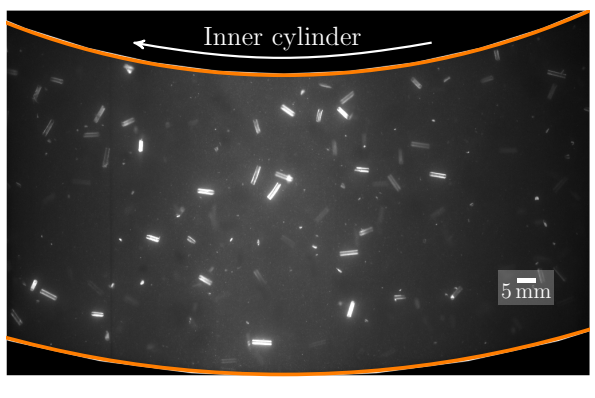

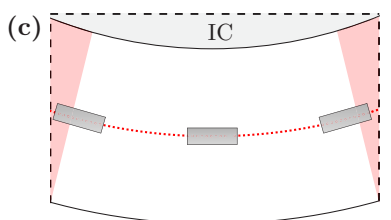

(d)

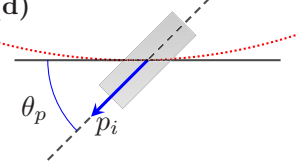

FIG. 1. (a) Schematic of the experimental apparatus (not to scale). The flow is confined between two concentric independently rotating cylinders with radii $r_{i}$ and $r_{o}$. Only the inner cylinder (IC) rotates with an angular velocity $\omega_{i}$. A mirror and a window in the bottom plate provide optical access to the $r-\theta$ plane for a high-speed camera. (b) A typical still image with the inner and outer cylinder highlighted in orange. The fibers (aspect ratio $\Lambda=5.3$ ) are clearly visible as white rods. $\operatorname{Re}_{i}=1.7 \times 10^{5}$ and $\alpha=0.05 \%$. (c) Schematic of the $r-\theta$ plane. The orientation of the particle $\theta_{p}$ is zero when it is aligned with the IC. Fibers with their center in the red areas are removed from all statistics. (d) Definition of the orientation $\theta_{p}$ and the orientation vector $p_{i}$ of a fiber. $\theta_{p}$ is measured with respect to the azimuthal direction and is defined positive in the counterclockwise direction. See Movies S1 and S2 in the Supplemental Material for typical recordings showing the movement and tracking of the fibers [43].

used, which is considered to be limited in its applicability to small sub-Kolmogorov scale $[23,24]$ particles, and they have a negligible particle Reynolds number [25].

In most practical situations, however, the suspended particles are not small, and they have a finite Reynolds number. Fully resolved numerical simulations, addressing the effect of fibers in turbulent channel flows, showed that finite-size effects lead to fiber-turbulence interactions that are significantly different from those of pointlike particles [26]. This can lead to an increased dissipation near the particle, and decreased dissipation in its wake. In such situations, no analytic expressions are available for the forces and torques acting on the particles. In general, it is considered that such finite-sized particles filter out the spatial and temporal flow fluctuations [27-35], and hence do not actively respond to the local gradients in the flow. Few experiments have explored this regime of finite-sized rodlike fibers in sheared turbulence.

In this Rapid Communication, we probe the dynamics of a suspension of millimetric rodlike fibers in a strongly turbulent Taylor-Couette (TC) flow [see Figs. 1(a) and 1(b)]. The reason for choosing this geometry is at least threefold: (i) It is a closed geometry, allowing for direct relationships between local and global quantities [36], (ii) there are no spatial transients, i.e., the turbulence intensity does not depend on the streamwise position as it does in channels and pipes, and (iii) it allows for high Reynolds numbers in a limited space [37]. All experiments are conducted in the Twente Turbulent Taylor-Couette $\left(\mathrm{T}^{3} \mathrm{C}\right)$ facility [38], which confines the flow between two concentric cylinders [see Fig. 1(a)]. The inner and outer cylinders radii are $r_{i}=0.2000 \mathrm{~m}$ and $r_{o}=0.2794 \mathrm{~m}$, respectively, giving a radius ratio of $\eta=r_{i} / r_{o}=0.716$ and a gap width $d=79.4 \mathrm{~mm}$. The height of the system is $L=0.927 \mathrm{~m}$, which results in an aspect ratio of $\Gamma=L / d=11.7$. We rotate the inner cylinder (IC) with angular velocity $\omega_{i}$ while the outer cylinder (OC) is kept at rest. The flow is seeded with rigid fibers of length $\ell=5.22 \pm 0.07 \mathrm{~mm}$, cut from a poly(methyl methacrylate) (PMMA) optical fiber of diameter $d_{p}=0.99 \pm 0.01 \mathrm{~mm}$ (aspect ratio $\Lambda=\ell / d_{p}=5.3 \pm 0.1$ ). The two-dimensional (2D) projection of the orientation angle on the radialazimuthal plane $\theta_{p}$ is defined to be zero when the fiber is aligned with the IC [Fig. 1(c)] and positive values are in the counterclockwise direction [Fig. 1(d)]. To minimize density effects, glycerol and water are mixed $1: 1$, giving a density ratio of $\rho_{p} / \rho_{\text {fluid }}=1210 \mathrm{~kg} \mathrm{~m}^{-3} / 1140 \mathrm{~kg} \mathrm{~m}^{-3}=1.06$. The dominant velocity is in the azimuthal direction. Velocities in the axial and radial directions are due 


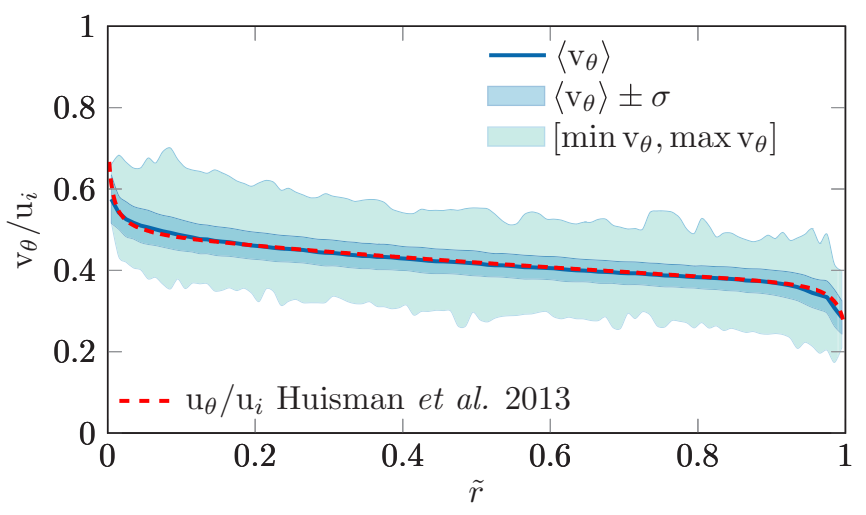

FIG. 2. Fiber velocity as a function of the dimensionless radius $\tilde{r}=\left(r-r_{i}\right) / d$ for $\operatorname{Re}_{i}=1.7 \times 10^{5}, \alpha=$ $0.05 \%$, and $\tilde{z}=z / L=0.24$. All velocities are normalized using the velocity of the IC $u_{i}$. For comparison, the azimuthal flow profile is included as a red dashed line. We find that the azimuthal velocity of the fibers is very close to the velocity of the flow.

to secondary flows and are approximately $5 \%$ of the azimuthal velocity. While the particles are free to rotate in all directions, the largest velocity gradient is in the radial direction, resulting in a rotation in the axial direction. For the flow under consideration, the control parameters are the Reynolds number $\operatorname{Re}_{i}=\omega_{i} r_{i}\left(r_{o}-r_{i}\right) / v$ and the volume fraction of the fibers $\alpha$. Here, $v$ is the the kinematic viscosity. $\operatorname{Re}_{i}$ is varied by changing $\omega_{i}$, resulting in a $\mathrm{Re}_{i}$ range from $8.3 \times 10^{4}$ to $2.5 \times 10^{5}$ which lies in the so-called ultimate regime [38-41] of turbulent Taylor-Couette flow, where both the bulk and boundary layers are turbulent. From the volume fraction of fibers $\alpha=0.025 \%$ to $\alpha=0.100 \%$, the suspensions we study are on the border of dilute and dense suspensions, which has either twoor four-way coupling [42]. To capture the orientation and velocities of the fibers, images in the radial-azimuthal plane are captured using a Photron SA-X2 high-speed camera. Illumination comes from a Litron LDY-303 pulsed laser and sheet optics, creating an approximately 2 -mm-thick plane. To improve the contrast, the fibers were coated using rhodamine-B making them fluorescent, and a bandpass filter was used isolate the fiber's signal. Figure 1(b) shows a typical captured image in which the IC and OC are highlighted [43]. A total amount of 64000 images per case $\left(\operatorname{Re}_{i}, \alpha, z / L\right)$ is captured and the positions and orientations of each of the fibers are extracted [see Figs. 1(c) and $1(\mathrm{~d})]$. These are then tracked over time, from which the velocity $v_{\theta}$ and angular velocity $\dot{\theta}_{p}$ can be determined. We find that the fibers distribute nearly homogeneously in the radial direction of the measurement volume. Moreover, we find that their azimuthal velocity, normalized using the velocity of the IC, $u_{i}$, closely follows the azimuthal velocity profile of the flow $u_{\theta}$ [44] (see Fig. 2). These fibers, therefore, do not show clustering or relative velocities, which seems surprising considering their rather large size. However, the absence of clustering can be expected, since the fibers are nearly neutrally buoyant $[28,45,46]$. Yet, this cannot explain the absence of relative velocities with the flow (see Fig. 2) we observe in our experiment. To explain this behavior, we calculate the Stokes number $\mathrm{St}_{K} \equiv \tau_{v} / \tau_{K}$, where $\tau_{v}=\frac{\ell^{2}}{3 \beta v}$ with $\beta=\frac{3 \rho_{f}}{2 \rho_{p}+\rho_{f}}$ the particle response time [47], and $\tau_{K}=\sqrt{\frac{v}{\epsilon}}$ is the Kolmogorov timescale. For our flow conditions we find that $\tau_{K}=[2.2 \mathrm{~ms}, 0.5 \mathrm{~ms}]$ and the Kolmogorov length scale $\eta_{K}=[113 \mu \mathrm{m}, 52.6 \mu \mathrm{m}]$, where each two values correspond to our lowest and highest Reynolds numbers $\operatorname{Re}_{i}=8.3 \times 10^{4}$ and $2.5 \times 10^{5}$, respectively. These values result then in $\mathrm{St}_{K}=[110.0,510.0]$, and size ratios $\ell / \eta_{K}=[44.0,95.0]$. This suggests that the fibers are large and highly inertial, and hence should filter out the flow fluctuations $[8,11,18,19,28,29]$. We therefore have to correct our previous Stokes number estimation as the relevant timescale is not given by $\tau_{K}$, but rather by the timescale $\tau_{\ell}$ of turbulent eddies comparable to the fiber size. $\tau_{\ell}=\left(\ell^{2} / \epsilon\right)^{1 / 3}$ [48], resulting in $\mathrm{St}_{\ell} \equiv \tau_{v} / \tau_{\ell}=[9.0,24.0]$. $\mathrm{St}_{\ell}$, though, assumes that the particles have a tiny Reynolds 


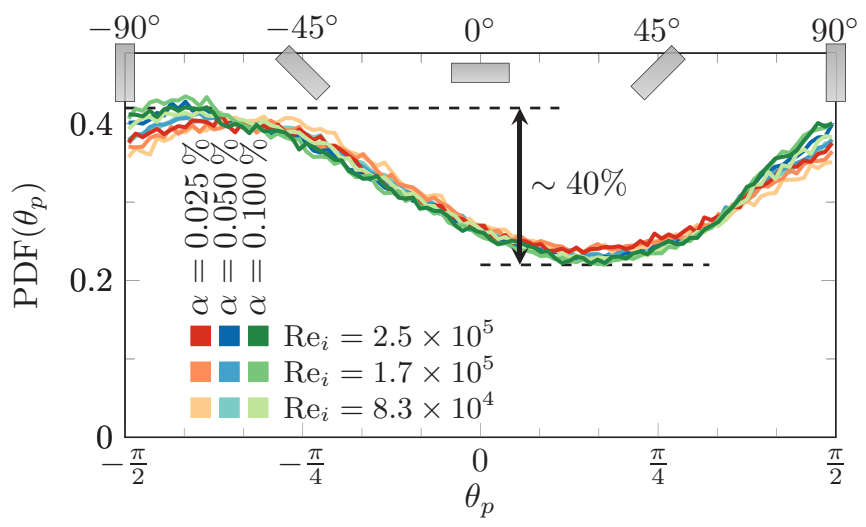

FIG. 3. PDF of the fiber orientation $\theta_{p}$ measured at $\tilde{z}=0.24$. Different $\alpha$ are indicated by different hues and different $\operatorname{Re}_{i}$ are shown with different shades. A representation of the fiber alignment is shown at the top of the figure. Independent of $\alpha$ and $\mathrm{Re}_{i}$ there is a clear preference for an alignment around $-0.38 \pi \pm$ $0.05 \pi\left(-68 \pm 9^{\circ}\right)$. A large $40 \%$ difference between the most and least probable orientation is observed.

numbers. Based on the liquid velocity fluctuations [28], we calculate the particle Reynolds number $\operatorname{Re}_{p}=\sigma\left(u_{\theta}\right) \ell / v=O\left(10^{3}\right)$, with $\sigma$ the standard deviation, which far exceeds the viscous flow limit. Based on these insights, we use a modified viscous timescale [47,49] for the particle $\tau_{p}$, which also takes into account the drag coefficient $C_{D}\left(\operatorname{Re}_{p}\right)$. Remarkably, the resulting Stokes number $\mathrm{St}_{p} \equiv \tau_{p} / \tau_{\ell}=[2.0,3.0]$, indicating that the fibers are only slightly inertial, which explains why they follow the flow field (at their length scale) quite accurately.

Next, we address the orientation statistics of the fibers in the flow. To check whether or not the fibers show any preferential alignment, we first look at the probability density function (PDF) of the orientation [see Fig. 1(d) for the definition] for various $\alpha$ and $\operatorname{Re}_{i}$ (see Fig. 3). We find that for all cases studied, the PDF of the orientation shows a preference for $\theta_{p}=-0.38 \pi \pm 0.05 \pi$ $\left(-68 \pm 9^{\circ}\right)$. Since Taylor-Couette flow [37] is known to have (turbulent) Taylor vortices [50,51] [relative positions shown in the right diagram of Fig. 4(b)], one might expect this preferential alignment to depend on the axial $(\tilde{z}=z / L)$ and radial $\left[\tilde{r}=\left(r-r_{i}\right) / d\right]$ positions of the fibers. We therefore provide PDFs conditioned on $\tilde{r}$, and perform additional measurements at several $\tilde{z}$ (see Fig. 4). Surprisingly, the preferential alignment around $\approx-0.38 \pi$ persists throughout the flow. We find nearly identical orientation PDFs for different $\operatorname{Re}_{i}$ and $\alpha$, and even at different $\tilde{r}$ and $\tilde{z}$. It is remarkable that a single preferential alignment value exists throughout the flow domain, despite the strong flow anisotropies and the finite size of the fibers.

In order to understand the preferential alignment of the fibers, we model their dynamics using a simplified model based on the equations by Jeffery [14], derived for ellipsoidal particles in a viscous fluid in the limit of small St and small $\mathrm{Re}_{p}$. Jeffery's equations in the noninertial limit are duplicated here,

$$
\dot{p}_{i}=\Omega_{i j} p_{j}+\frac{\Lambda^{2}-1}{\Lambda^{2}+1}\left(S_{i j} p_{j}-p_{i} p_{k} S_{k l} p_{l}\right)
$$

where $p_{i}$ is the orientation vector [see Fig. 1(d)], $\Omega_{i j}$ is the vorticity tensor $\Omega_{i j}=\frac{1}{2}\left(\frac{\partial u_{i}}{\partial x_{j}}-\frac{\partial u_{j}}{\partial x_{i}}\right)=$ $-\epsilon_{i j k} \omega_{k}$, where $\epsilon_{i j k}$ is the Levi-Civita symbol in three dimensions (3D), $\omega_{k}$ is the vorticity vector, $\Lambda$ is the aspect ratio of the particle, and $S_{i j}$ is the strain-rate tensor $S_{i j}=\frac{1}{2}\left(\frac{\partial u_{i}}{\partial x_{j}}+\frac{\partial u_{j}}{\partial x_{i}}\right)$. We model a stochastic mean-field process by assuming that the fibers are initially randomly oriented by the turbulent fluctuations every time interval $\sim O\left(\tau_{\ell}\right)$. Next, we model the flow seen by the fiber as a simple shear flow, with a mean shear rate equal to that in the bulk of the turbulent Taylor-Couette flow, i.e., $\dot{\gamma}=\left\langle\partial u_{\theta} / \partial r\right\rangle_{\tilde{r} \in[0.25,0.75]}$ from Fig. 2. We integrate Eqs. (1) for all initial conditions 

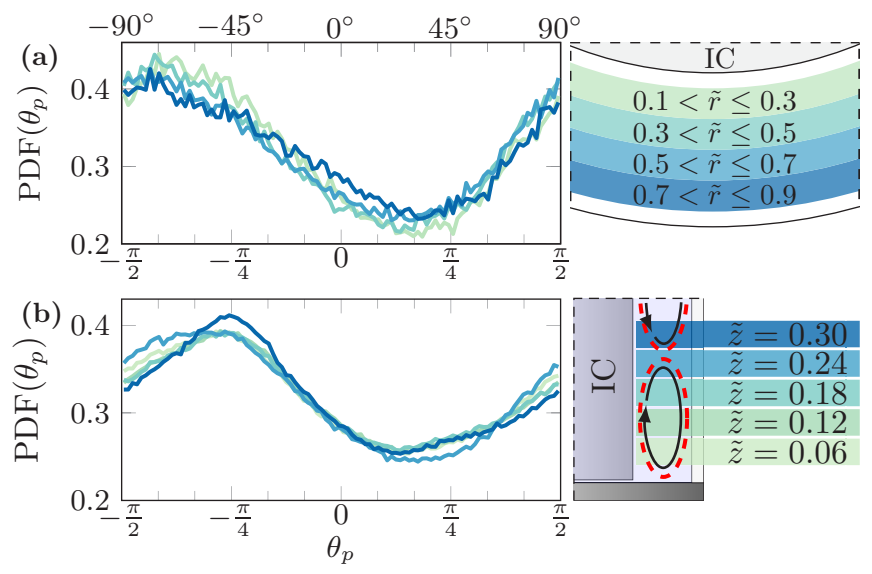

FIG. 4. (a) PDF of the fiber orientation $\theta_{p}$ at various radial bins, indicated by different colors. $\alpha$ is fixed to $0.05 \%, \operatorname{Re}_{i}=2.5 \times 10^{5}$, and the measurement is performed at $\tilde{z}=0.24$. (b) Axial dependence of the PDF of $\theta_{p}$, indicated by different colors. For these measurements, $\alpha=0.05 \%$ and $\operatorname{Re}_{i}=8.3 \times 10^{4}$. The diagram on the right indicates the position of the weak vortical structures [50,51]. The distribution is found to be nearly independent of radial and axial positions, and all show similar alignment.

$p_{i}(t=0)=\sin \left(\frac{\pi i}{2}-\theta\right)$ for $\theta \in[-\pi / 2, \pi / 2]$ over a variety of timescales $t \in\left[0, C \tau_{\ell}\right]$ where $C$ is a dimensionless constant of $O(1)$, to obtain $p_{i}(t)$ for every initial condition. These evolutions of $p_{i}(t)$ are converted to $\theta(t)$ using the definition given in Fig. 1(d), aggregated, and binned to calculate the PDF of $\theta$ (see Fig. 5). The PDF shape predicted by this simplified model is remarkably similar to our experimental observations. The preferred orientation (peak) calculated from Jeffery's equation is approximately $-0.27 \pi\left(\approx 50^{\circ}\right)$, which is close to the measured value of $-0.38 \pi\left(\approx 68^{\circ}\right)$. The shift of approximately $15^{\circ}$ can be explained by a slight inertial effect: As the particle goes around the inner cylinder, it continuously adapts to the local orientation of the flow. We believe that this discrepancy is likely due to a lag towards this preferred alignment. Also, the turbulent fluctuations, which are not considered in this model, can cause an offset towards the preferred alignment. The amplitude of the measured PDF is close to the calculation with the integration time being $2 \tau_{\ell}$, which measures the timescale of rotation of a noninertial fiber in the bulk of the flow.

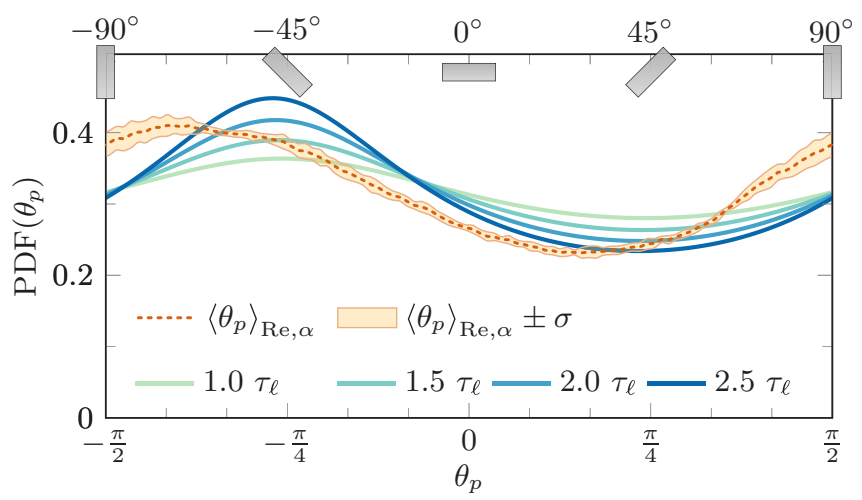

FIG. 5. Averaged PDF of the experimentally found fiber orientation (dashed) compared to the alignment found from integrating Jeffery's equations (solid lines). The legend indicates the integration timescale as multiples of $\tau_{\ell}$. 


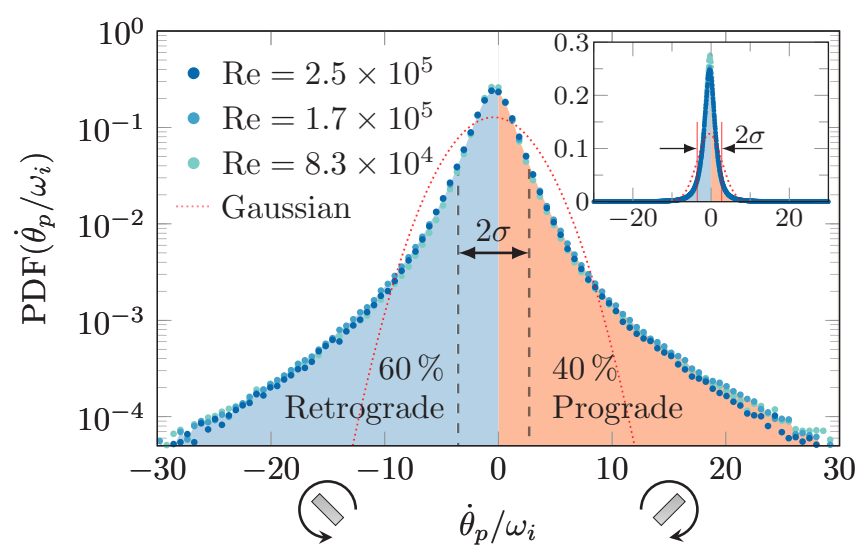

FIG. 6. PDF of the rotation rate of the fibers for $\alpha=0.05 \%$ and $z / L=0.24$. Rotational velocities are normalized using the angular velocity of the IC. The PDF is independent of $\operatorname{Re}_{i}$ and shows a slight preference for retrograde rotation (blue). Note that the icons hold for clockwise $(\mathrm{CW})$ rotation of the inner cylinder. The mean rotation is $\left\langle\dot{\theta}_{p} / \omega_{i}\right\rangle \approx-0.42$ with a standard deviation of $\sigma\left(\dot{\theta}_{p} / \omega_{i}\right)=3.13$, which reveals that a large number of fibers rotate much faster than the inner cylinder. For comparison, a Gaussian distribution with the same mean and variance is added. The skewness and kurtosis are found to lie in the range [-0.14, 0.24] and $[34,40]$, respectively. The inset shows the same data on a linear scale.

The noninertial approach for orientation modeling is reasonable, since typically the rotational Stokes number $\mathrm{St}_{r} \sim O\left(0.1 \mathrm{St}_{p}\right)$ for long prolate ellipsoids [22]. Nevertheless, slight differences between the calculation and experimental results (seen in Fig. 5) are expected, since our fibers are not truly in the $\mathrm{St}_{r} \rightarrow 0$ limit [52]. The PDF of the fiber rotation rate at different $\mathrm{Re}_{i}$ is shown in Fig. 6 (the inset shows the same data on linear scale). The PDF of the normalized rotation rate is found to be independent of the Reynolds number. Due to the mean shear in the bulk of the flow, it has a slight preference for retrograde rotation (rotation in the opposite direction of the IC) with $60 \%$ probability. We notice that the peak of the PDF is located at $\left\langle\dot{\theta_{p}} / \omega_{i}\right\rangle \approx-0.42$, which is comparable to the mean vorticity in the bulk of the flow. The standard deviation is $\sigma\left(\dot{\theta}_{p} / \omega_{i}\right)=3.13$, which reveals that a large number of fibers rotate much faster than the inner cylinder. What is really remarkable is the strong intermittency of the PDF with tails extending beyond $\pm 30 \omega_{i}$, which occurs despite the large size of the fibers. We find a skewness of the angular velocity between [-0.14, 0.24]. The kurtosis of the angular velocity lies in the range $[34,40]$, which is much larger than the kurtosis of spheres of similar size ratios [53-55]. This can be attributed to the fact that for elongated ellipsoids the rotational inertia is typically much lower than the rotational inertia of similar-sized spheres [22]. Furthermore, the length of the fibers is of $O\left(100 \eta_{K}\right)$, so the two ends of the fibers can therefore experience extremely high instantaneous velocity differences due to the intermittent nature of the turbulent velocity fluctuations. These instantaneous velocity differences can create high torques on the fiber, resulting in violent rotational intermittency.

To summarize, we report on the statistics of translation and rotation of finite-sized fibers in a strongly sheared turbulent flow. The fibers tend to follow the flow almost perfectly, despite their large size. This adherence to the flow can be explained by considering the turbulent dynamic time at the scale of the fiber, compensated by effects of nonlinear drag at the finite Reynolds number of the fiber, yielding a Stokes number estimate that is just above unity. For the fiber orientation statistics, while it was often hypothesized that no systematic alignment would be possible in such highly turbulent flows with very strong liquid fluctuations, in this canonical TC flow geometry, we show that fibers do align with an angle of $-0.38 \pi \pm 0.05 \pi\left(-68 \pm 9^{\circ}\right)$ with respect to the IC wall. The difference between the most and least preferred alignment is dramatic, namely, 40\%. This alignment is similar for all tested Reynolds numbers $\left(\operatorname{Re}_{i}\right)$, fiber volume fractions $(\alpha)$, and spatial coordinates. 
We model the fiber orientation statistics using Jeffery's equations, which provides a fair estimate of the shape of the alignment PDFs. Additionally, it is found that the fiber angular velocity shows extremely high intermittency with instantaneous rotation rates much larger than that of the rotating cylinder. Thus, in a number of ways, finite-sized (millimetric) fibers behave remarkably similar to tiny particles in turbulence $[11,27]$, extending the possibilities of the point-particle approach to model large anisotropic particles in turbulence.

We thank Eric Climent, Enrico Calzavarini, Vamsi Spandan, Dominik Krug, Jelle Will, Pim Bullee, and Arne te Nijenhuis for various stimulating discussions, and Gert-Wim Bruggert and Martin Bos for technical support. This work was funded by the Natural Science Foundation of China under Grants No. 91852202 and No. 11861131005, the Netherlands Organisation for Scientific Research (NWO) under VIDI Grant No. 13477, STW, FOM, and MCEC.

[1] F. Lundell, L. D. Söderberg, and P. H. Alfredsson, Fluid mechanics of papermaking, Annu. Rev. Fluid Mech. 43, 195 (2011).

[2] L. Sabban, A. Cohen, and R. van Hout, Temporally resolved measurements of heavy, rigid fibre translation and rotation in nearly homogeneous isotropic turbulence, J. Fluid Mech. 814, 42 (2017).

[3] K. Vercruysse, R. C. Grabowski, and R. J. Rickson, Suspended sediment transport dynamics in rivers: Multi-scale drivers of temporal variation, Earth-Sci. Rev. 166, 38 (2017).

[4] D. Lopez and E. Guazzelli, Inertial effects on fibers settling in a vortical flow, Phys. Rev. Fluids 2, 024306 (2017).

[5] J. E. Butler and B. Snook, Microstructural dynamics and rheology of suspensions of rigid fibers, Annu. Rev. Fluid Mech. 50, 299 (2018).

[6] M. Daghooghi and I. Borazjani, The influence of inertia on the rheology of a periodic suspension of neutrally buoyant rigid ellipsoids, J. Fluid Mech. 781, 506 (2015).

[7] M. Wilkinson, V. Bezuglyy, and B. Mehlig, Fingerprints of random flows? Phys. Fluids 21, 043304 (2009).

[8] S. Parsa, J. S. Guasto, M. Kishore, N. T. Ouellette, J. P. Gollub, and G. A. Voth, Rotation and alignment of rods in two-dimensional chaotic flow, Phys. Fluids 23, 043302 (2011).

[9] A. Pumir and M. Wilkinson, Orientation statistics of small particles in turbulence, New J. Phys. 13, 093030 (2011).

[10] S. Parsa, E. Calzavarini, F. Toschi, and G. A. Voth, Rotation Rate of Rods in Turbulent Fluid Flow, Phys. Rev. Lett. 109, 134501 (2012).

[11] G. A. Voth and A. Soldati, Anisotropic particles in turbulence, Annu. Rev. Fluid Mech. 49, 249 (2017).

[12] R. Ni, N. T. Ouellette, and G. A. Voth, Alignment of vorticity and rods with Lagrangian fluid stretching in turbulence, J. Fluid Mech. 743, R3 (2014).

[13] D. Vincenzi, Orientation of non-spherical particles in an axisymmetric random flow, J. Fluid Mech. 719, 465 (2013).

[14] G. B. Jeffery, The motion of ellipsoidal particles immersed in a viscous fluid, Proc. R. Soc. London, Ser. A 102, 161 (1922).

[15] P. Butler, Shear induced structures and transformations in complex fluids, Curr. Opin. Colloid Interface Sci. 4, 214 (1999).

[16] H. Zhang, G. Ahmadi, F.-G. Fan, and J. B. McLaughlin, Ellipsoidal particles transport and deposition in turbulent channel flows, Int. J. Multiphase Flow 27, 971 (2001).

[17] P. H. Mortensen, H. I. Andersson, J. J. J. Gillissen, and B. J. Boersma, Dynamics of prolate ellipsoidal particles in a turbulent channel flow, Phys. Fluids 20, 093302 (2008).

[18] C. Marchioli, M. Fantoni, and A. Soldati, Orientation, distribution, and deposition of elongated, inertial fibers in turbulent channel flow, Phys. Fluids 22, 033301 (2010). 
[19] C. Marchioli and A. Soldati, Rotation statistics of fibers in wall shear turbulence, Acta Mech. 224, 2311 (2013).

[20] N. R. Challabotla, L. Zhao, and H. I. Andersson, Orientation and rotation of inertial disk particles in wall turbulence, J. Fluid Mech. 766, R2 (2015).

[21] F. Zhao, W. K. George, and B. G. M. van Wachem, Four-way coupled simulations of small particles in turbulent channel flow: The effects of particle shape and Stokes number, Phys. Fluids 27, 083301 (2015).

[22] L. Zhao, N. R. Challabotla, H. I. Andersson, and E. A. Variano, Rotation of Nonspherical Particles in Turbulent Channel Flow, Phys. Rev. Lett. 115, 244501 (2015).

[23] A. N. Kolmogorov, The local structure of turbulence in incompressible viscous fluid for very large Reynolds numbers, Dokl. Akad. Nauk. SSSR 30, 301 (1941).

[24] A. N. Kolmogorov, On degeneration of isotropic turbulence in an incompressible viscous liquid, Dokl. Akad. Nauk. SSSR 31, 538 (1941).

[25] M. R. Maxey and J. J. Riley, Equation of motion for a small rigid sphere in a nonuniform flow, Phys. Fluids 26, 883 (1983).

[26] M. Do-Quang, G. Amberg, G. Brethouwer, and A. V. Johansson, Simulation of finite-size fibers in turbulent channel flows, Phys. Rev. E 89, 013006 (2014).

[27] F. Toschi and E. Bodenschatz, Lagrangian properties of particles in turbulence, Annu. Rev. Fluid Mech. 41, 375 (2009).

[28] E. Calzavarini, R. Volk, M. Bourgoin, E. Lévêque, J.-F. Pinton, and F. Toschi, Acceleration statistics of finite-sized particles in turbulent flow: The role of Faxén forces, J. Fluid Mech. 630, 179 (2009).

[29] J. Bec, L. Biferale, A. S. Lanotte, A. Scagliarini, and F. Toschi, Turbulent pair dispersion of inertial particles, J. Fluid Mech. 645, 497 (2010).

[30] G. Bellani and E. A. Variano, Slip velocity of large neutrally buoyant particles in turbulent flows, New J. Phys. 14, 125009 (2012).

[31] G. Bellani, M. L. Byron, A. G. Collignon, C. R. Meyer, and E. A. Variano, Shape effects on turbulent modulation by large nearly neutrally buoyant particles, J. Fluid Mech. 712, 41 (2012).

[32] V. Mathai, V. N. Prakash, J. Brons, C. Sun, and D. Lohse, Wake-Driven Dynamics of Finite-Sized Buoyant Spheres in Turbulence, Phys. Rev. Lett. 115, 124501 (2015).

[33] E. Alméras, V. Mathai, D. Lohse, and C. Sun, Experimental investigation of the turbulence induced by a bubble swarm rising within incident turbulence, J. Fluid Mech. 825, 1091 (2017).

[34] D. Bakhuis, R. A. Verschoof, V. Mathai, S. G. Huisman, D. Lohse, and C. Sun, Finite-sized rigid spheres in turbulent Taylor-Couette flow: Effect on the overall drag, J. Fluid Mech. 850, 246 (2018).

[35] V. Mathai, S. G. Huisman, C. Sun, D. Lohse, and M. Bourgoin, Dispersion of Air Bubbles in Isotropic Turbulence, Phys. Rev. Lett. 121, 054501 (2018).

[36] B. Eckhardt, S. Grossmann, and D. Lohse, Torque scaling in turbulent Taylor-Couette flow between independently rotating cylinders, J. Fluid Mech. 581, 221 (2007).

[37] S. Grossmann, D. Lohse, and C. Sun, High-Reynolds number Taylor-Couette turbulence, Annu. Rev. Fluid Mech. 48, 53 (2016).

[38] D. P. M. van Gils, G.-W. Bruggert, D. P. Lathrop, C. Sun, and D. Lohse, The Twente turbulent TaylorCouette $\left(\mathrm{T}^{3} \mathrm{C}\right)$ facility: Strongly turbulent (multiphase) flow between independently rotating cylinders, Rev. Sci. Instrum. 82, 025105 (2011).

[39] D. P. Lathrop, J. Fineberg, and H. L. Swinney, Turbulent Flow Between Concentric Rotating Cylinders at Large Reynolds Number, Phys. Rev. Lett. 68, 1515 (1992).

[40] S. G. Huisman, D. P. M. van Gils, S. Grossmann, C. Sun, and D. Lohse, Ultimate Turbulent Taylor-Couette Flow, Phys. Rev. Lett. 108, 024501 (2012).

[41] R. Ostilla-Mónico, E. P. van der Poel, R. Verzicco, S. Grossmann, and D. Lohse, Exploring the phase diagram of fully turbulent Taylor-Couette flow, J. Fluid Mech. 761, 1 (2014).

[42] S. Elghobashi, On predicting particle-laden turbulent flows, Appl. Sci. Res. 52, 309 (1994).

[43] See Supplemental Material at http://link.aps.org/supplemental/10.1103/PhysRevFluids.4.072301 for a video of a typical recording (Movie S1) and for a video on movement and tracking of the fibers (Movie S2). 
[44] S. G. Huisman, S. Scharnowski, C. Cierpka, C. J. Kähler, D. Lohse, and C. Sun, Logarithmic Boundary Layers in Strong Taylor-Couette Turbulence, Phys. Rev. Lett. 110, 264501 (2013).

[45] E. Calzavarini, M. Cencini, D. Lohse, and F. Toschi, Quantifying Turbulence-Induced Segregation of Inertial Particles, Phys. Rev. Lett. 101, 084504 (2008).

[46] L. Fiabane, R. Zimmermann, R. Volk, J.-F. Pinton, and M. Bourgoin, Clustering of finite-size particles in turbulence, Phys. Rev. E 86, 035301(R) (2012).

[47] N. M. Qureshi, M. Bourgoin, C. Baudet, A. Cartellier, and Y. Gagne, Turbulent Transport of Material Particles: An Experimental Study of Finite Size Effects, Phys. Rev. Lett. 99, 184502 (2007).

[48] H. Xu and E. Bodenschatz, Motion of inertial particles with size larger than Kolmogorov scale in turbulent flows, Physica D (Amsterdam) 237, 2095 (2008).

[49] R. Clift, J. R. Grace, M. E. Weber, and M. F. Weber, Bubbles, Drops, and Particles (Academic, New York, 1978).

[50] S. G. Huisman, R. C. A. van der Veen, C. Sun, and D. Lohse, Multiple states in highly turbulent TaylorCouette flow, Nat. Commun. 5, 3820 (2014).

[51] R. C. A. van der Veen, S. G. Huisman, O.-Y. Dung, H. L. Tang, C. Sun, and D. Lohse, Exploring the phase space of multiple states in highly turbulent Taylor-Couette flow, Phys. Rev. Fluids 1, 024401 (2016).

[52] N. R. Challabotla, C. Nilsen, and H. I. Andersson, On rotational dynamics of inertial disks in creeping shear flow, Phys. Lett. A 379, 157 (2015).

[53] R. Zimmermann, Y. Gasteuil, M. Bourgoin, R. Volk, A. Pumir, and J.-F. Pinton, Rotational Intermittency and Turbulence Induced Lift Experienced by Large Particles in a Turbulent Flow, Phys. Rev. Lett. 106, 154501 (2011).

[54] V. Mathai, M. W. M. Neut, E. P. van der Poel, and C. Sun, Translational and rotational dynamics of a large buoyant sphere in turbulence, Exp. Fluids 57, 51 (2016).

[55] V. Mathai, X. Zhu, C. Sun, and D. Lohse, Flutter to tumble transition of buoyant spheres triggered by rotational inertia changes, Nat. Commun. 9, 1792 (2018). 